\title{
Discriminator logics \\ (Research announcement)
}

\author{
M. SPINKS \\ Department of Philosophy \\ University of Cagliari \\ Via Is Mirrionis 1 \\ 09123 Cagliari \\ Italy \\ mspinksau@yahoo.com.au
}

\author{
R. J. BignalL \\ Sunway University \\ 5 Jalan Universiti \\ Bandar Sunway \\ 46150 Petaling Jaya \\ Selangor Darul Ehsan \\ Malaysia \\ rjbignall@gmail.com
}

\author{
R. VEROFF \\ Department of Computer Science \\ University of New Mexico \\ Albuquerque NM 87131 \\ U.S.A. \\ veroff@cs.unm.edu
}

\begin{abstract}
A discriminator logic is the 1 -assertional logic of a discriminator variety $\mathcal{V}$ having two constant terms $\mathbf{0}$ and $\mathbf{1}$ such that $\mathcal{V} \models \mathbf{0} \approx \mathbf{1}$ iff every member of $\mathcal{V}$ is trivial. Examples of such logics abound in the literature. The main result of this research announcement asserts that a certain non-Fregean deductive system SBPC, which closely resembles the classical propositional calculus, is canonical for the class of discriminator logics in the sense that any discriminator logic $\mathbf{S}$ can be presented (up to definitional equivalence) as an axiomatic extension of SBPC by a set of extensional logical connectives taken from the language of $\mathbf{S}$. The results outlined in this research announcement are extended to several generalisations of the class of discriminator logics in the main work.
\end{abstract}




\section{Introduction}

The ternary discriminator on a set $A$ is the function $t: A^{3} \rightarrow A$ defined for all $a, b, c \in A$ by [13, Chapter IV $\S 9]$ :

$$
t(a, b, c):= \begin{cases}c & \text { if } a=b \\ a & \text { otherwise }\end{cases}
$$

A discriminator variety is a variety $\mathcal{V}$ for which there exists a ternary term $t(x, y, z)$ of $\mathcal{V}$ that realises the ternary discriminator on every subdirectly irreducible member of $\mathcal{V}$. The term $t(x, y, z)$ is called a discriminator term for $\mathcal{V}$. A variety $\mathcal{V}$ with constant terms $\mathbf{0}$ and $\mathbf{1}$ is a $(\mathbf{0}, \mathbf{1})$-discriminator variety if $\mathcal{V}$ is a discriminator variety, and moreover, $\Theta^{\mathbf{A}}\left(\mathbf{0}^{\mathbf{A}}, \mathbf{1}^{\mathbf{A}}\right)=A \times A$ for any $\mathbf{A} \in \mathcal{V}$.

The logics inherent in $(\mathbf{0}, \mathbf{1})$-discriminator varieties encompass many wellknown and important deductive systems that have previously been considered in the literature, as well as numerous other interesting but less well known logics. For specific examples, see Section 2. The main result of this research announcement asserts that, up to definitional equivalence, all such 'discriminator logics' can be presented as axiomatic expansions of a certain non-Fregean analogue of the classical propositional calculus. See Theorem 4.1 below.

Details of all results reported in this announcement, including proofs, may be found in the monograph [37] in preparation.

\section{Discriminator logics}

Following [7], a deductive system is a pair $\langle\Lambda, \vdash\rangle$, where $\Lambda$ is an algebraic language and $\vdash$ is a finitary and substitution-invariant consequence relation over $\Lambda$. Given a deductive system $\langle\Lambda, \vdash\rangle$, the set of all $\Lambda$-formulas is denoted by $\mathrm{Fm}_{\Lambda}$.

Let $\mathcal{V}$ be a variety with a constant term 1 over a language $\Lambda$. The 1-assertional logic of $\mathcal{V}$, in symbols $S(\mathcal{V}, \mathbf{1})$, is the deductive system $\left\langle\Lambda, \vdash_{S(\mathcal{V}, \mathbf{1})}\right\rangle$ whose consequence relation $\vdash_{S(\mathcal{V}, \mathbf{1})}$ is determined by the equivalence [10, Definition 3.1.1]

$$
\Gamma \vdash_{S(\mathcal{V}, \mathbf{1})} \varphi \text { iff } \quad\{\psi \approx \mathbf{1}: \psi \in \Gamma\} \models_{\mathcal{V}} \varphi \approx \mathbf{1}
$$

The 'logics inherent in $(\mathbf{0}, \mathbf{1})$-discriminator varieties' of the informal remarks of the introduction are exactly the $\mathbf{1}$-assertional logics of $(\mathbf{0}, \mathbf{1})$-discriminator varieties. 
A deductive system $\mathbf{S}$ is a discriminator logic if $\mathbf{S}=S(\mathcal{V}, \mathbf{1})$ for a $(\mathbf{0}, \mathbf{1})$-discriminator variety $\mathcal{V}$. Examples of discriminator logics abound in the literature and include:

- CPC, the classical propositional calculus.

- The normal modal logic S5 ("Gödel style") [9].

- The $n$-dimensional cylindric logics.

- The $n$-valued Post logics.

- The $n$-valued Łukasiewicz logics.

- Basic fuzzy logic [26] with Baaz delta [1].

- The $n$-valued Łukasiewicz-Moisil logics [3].

- The $n$-valued symmetrical modal propositional calculi of $[29,30]$.

- The logic LPF of the Vienna Development Method [2, 31].

- The tetravalent modal logic of [23].

- The dreiwertige Logik der Sprache of [5] at the propositional level.

- The Heyting-Wajsberg fuzzy logic of $[15,16]$.

- The R5-calculus and the logic PC of [21].

- The modal-type orthomodular logic of $[19,20]$.

- Linear tense logics [32].

This list is not exhaustive.

\section{The skew Boolean propositional calculus}

Let $\Lambda[\mathbf{S B P C}]$ denote the language consisting of the binary logical connectives $\wedge, \vee, \Rightarrow$, and $\rightarrow$ (respectively, the conjunction, disjunction, strong implication, and weak implication signs) and the unary logical connective $\neg$ (the negation sign). The skew Boolean propositional calculus, in symbols 
SBPC, is the deductive system over $\Lambda[$ SBPC $]$ presented by the following set $\Sigma[$ SBPC $]$ of axioms and inference rules:

$$
\begin{aligned}
& \vdash \varphi \Rightarrow(\psi \Rightarrow \varphi) \\
& \vdash(\varphi \Rightarrow(\psi \Rightarrow \chi)) \Rightarrow((\varphi \Rightarrow \psi) \Rightarrow(\varphi \Rightarrow \chi)) \\
& \vdash((\varphi \Rightarrow \psi) \Rightarrow \varphi) \Rightarrow \varphi \\
& \vdash \varphi \Rightarrow(\psi \rightarrow \varphi) \\
& \vdash(\varphi \rightarrow(\psi \rightarrow \chi)) \Rightarrow((\varphi \rightarrow \psi) \rightarrow(\varphi \rightarrow \chi)) \\
& \vdash((\varphi \rightarrow \psi) \rightarrow \varphi) \Rightarrow \varphi \\
& \vdash((\varphi \Rightarrow \psi) \rightarrow \psi) \Rightarrow((\psi \Rightarrow \varphi) \rightarrow \varphi) \\
& \vdash(\varphi \Rightarrow \psi) \rightarrow(\varphi \rightarrow \psi) \\
& \vdash \varphi \rightarrow(\varphi \vee \psi) \\
& \vdash \psi \Rightarrow(\varphi \vee \psi) \\
& \vdash(\varphi \rightarrow \chi) \rightarrow((\psi \rightarrow \chi) \rightarrow((\varphi \vee \psi) \rightarrow \chi)) \\
& \vdash(\varphi \wedge \psi) \Rightarrow \varphi \\
& \vdash(\varphi \wedge \psi) \rightarrow \psi \\
& \vdash(\varphi \Rightarrow \psi) \Rightarrow((\varphi \Rightarrow \chi) \Rightarrow(\varphi \Rightarrow(\psi \wedge \chi))) \\
& \vdash(\varphi \rightarrow \psi) \rightarrow((\varphi \Rightarrow \neg \psi) \Rightarrow \neg \varphi) \\
& \vdash \neg \varphi \rightarrow(\varphi \rightarrow \psi) \\
& \varphi, \varphi \rightarrow \psi \vdash \psi
\end{aligned}
$$

Observe that, by $(3.3)$ and $\left(\mathrm{MP}_{\rightarrow}\right)$, modus ponens for $\Rightarrow$ is a derived rule of inference of SBPC.

Let $\Lambda[\mathbf{C P C}]$ denote the language consisting of the binary logical connectives $\cap, \cup$, and $\supset$ (respectively, the conjunction, disjunction, and implication signs) and the unary logical connective $\sim$ (the negation sign). Further, let $\Sigma[\mathbf{C P C}]$ be a standard presentation (such as that of [9, Section 2.2.1]) of CPC over the language $\Lambda[\mathbf{C P C}]$.

For any $\{\supset\}$-formula $\varphi$, let $\varphi[\Rightarrow]$ and $\varphi[\rightarrow]$ denote the formulas obtained from $\varphi$ on replacing every occurrence of $\supset$ in $\varphi$ by $\Rightarrow$ and by $\rightarrow$ respectively. For any set of $\{\supset\}$-formulas $\Gamma$, let $\Gamma[\Rightarrow]:=\{\varphi[\Rightarrow]: \varphi \in \Gamma\}$ and $\Gamma[\rightarrow]:=$ $\{\varphi[\rightarrow]: \varphi \in \Gamma\}$. Then for any set of $\{\supset\}$-formulas $\Gamma \cup\{\varphi\}, \Gamma \vdash_{\mathbf{C P C}} \varphi$ iff $\Gamma[\Rightarrow] \vdash_{\text {SBPC }} \varphi[\Rightarrow]$ iff $\Gamma[\rightarrow] \vdash_{\text {SBPC }} \varphi[\rightarrow]$. In contrast, $\vdash_{\mathbf{C P C}}((\varphi \supset \psi) \supset$ $\psi) \supset((\psi \supset \varphi) \supset \varphi)$ but $\nvdash_{\text {SBPC }}((\varphi \rightarrow \psi) \rightarrow \psi) \Rightarrow((\psi \rightarrow \varphi) \rightarrow \varphi)$. For more details on the relationship between $\Rightarrow, \rightarrow$, and material implication, 
see $[27,28]$ or [37].

Let SBPC $^{F}$ denote the axiomatic extension of SBPC by the (implicational) Fregean axiom

$$
\vdash(\varphi \rightarrow \psi) \Rightarrow(\varphi \Rightarrow \psi)
$$

The (implicational) Fregean axiom arises from the 'unsymmetrisation' of the Fregean axiom

$$
\vdash(\varphi \subset \supset \psi) \equiv(\varphi \equiv \psi)
$$

Here $\subset \supset$ denotes the (material) biconditional and $\equiv$ denotes the (primitive or derived) binary logical connective of logical identity. The logical connective $\equiv$ arises naturally in the context of Suszko's sentential calculus with identity SCI $[38,39,40]$, which augments CPC with $\equiv$ in order that the distinction between logical identity and logical equivalence may be realised. The Fregean axiom is characteristic of Fregean logics, namely, those deductive systems for which the formulas $\varphi \equiv \psi$ and $\varphi \subset \supset \psi$ are synonymous in the sense of Smiley [35]. The paradigm of a Fregean logic [resp. non-Fregean logic] is CPC [resp. SCI]. For a discussion and references, see [17, 18].

The next result asserts that, to within definitional equivalence, CPC is the axiomatic extension of SBPC by the (implicational) Fregean axiom. Hence SBPC is a non-Fregean generalisation of CPC. For the precise relationship between SBPC and SCI, see [37].

\section{Proposition 3.1.}

1. The map $\delta: \Lambda[\mathbf{S B P C}] \rightarrow \mathrm{Fm}_{\Lambda[\mathbf{C P C}]}$ defined by

$$
\begin{array}{rlrl}
p \wedge q \mapsto p \cap q & & p \rightarrow q \mapsto p \supset q \\
p \vee q & \mapsto p \cup q & & p \Rightarrow q \mapsto p \supset q \\
\neg p & \mapsto \sim p & &
\end{array}
$$

is an interpretation of $\mathbf{S B P C}^{F}$ in $\mathbf{C P C}$.

2. The map $\epsilon: \Lambda[\mathbf{C P C}] \rightarrow \mathrm{Fm}_{\Lambda[\mathbf{S B P C}]}$ defined by

$$
\begin{array}{ll}
p \cap q \mapsto p \wedge q & p \supset q \mapsto p \rightarrow q \\
p \cup q \mapsto p \vee q & \sim p \mapsto \neg p
\end{array}
$$

is an interpretation of $\mathbf{C P C}$ in $\mathbf{S B P C}{ }^{F}$.

3. The interpretations $\delta$ and $\epsilon$ are mutually inverse. 
Hence the deductive systems $\mathbf{S B P C}^{F}$ and $\mathbf{C P C}$ are definitionally equivalent.

Let $\mathcal{G} \mathcal{D}_{(\mathbf{0 , 1})}$ denote the variety generated by the class of all algebras $\mathbf{A}:=$ $\langle A ; t, 0,1\rangle$ of type $\langle 3,0,0\rangle$, where $t$ is the ternary discriminator on $A$ and 0 and 1 are nullary operations such that $\Theta^{\mathbf{A}}(0,1)=A \times A$. A proof of the following theorem may be extracted from results of [4] and [41].

Theorem 3.2. The deductive system $S(\mathcal{G D}(\mathbf{0 , 1}), \mathbf{1})$ is, up to definitional equivalence, precisely SBPC.

The notion of definitional equivalence exploited above and throughout this announcement is due to [25]. For deductive systems that are strongly and regularly algebraisable (in the sense of [18]), it is an analogue of the well known notion of term equivalence [34] for varieties. For details, see [36].

Necessary conditions to be satisfied by any reasonable notion of definitional equivalence are postulated in [14]. There, it is asserted that deductive systems $\mathbf{S}_{1}$ and $\mathbf{S}_{2}$ are definitionally equivalent if there exist

- mutually inverse syntactic translations, uniform in the sense of [14], mapping formulas of $\mathbf{S}_{1}$ to formulas of $\mathbf{S}_{2}$ and conversely, and

- mutually inverse lattice isomorphisms between the lattices of theories of $\mathbf{S}_{1}$ and $\mathbf{S}_{2}$.

These stipulations are met by the notion of definitional equivalence used in this work.

\section{The main result}

Let $\mathcal{F}$ be a language with $\mathcal{F} \cap \Lambda[\mathbf{S B P C}]=\emptyset$. Let $\mathbf{S B P C}[\mathcal{F}]$ denote the expansion of SBPC to the language $\Lambda[\mathbf{S B P C}] \cup \mathcal{F}$ obtained by adjoining, for each $n$-ary logical connective $f \in \mathcal{F}$ and each $1 \leq i \leq n$, the compatibility axioms

$$
\vdash(\varphi \Rightarrow \psi) \rightarrow\left((\psi \Rightarrow \varphi) \rightarrow\left(f\left(\vec{\chi}_{i-1}, \varphi, \vec{\chi}_{i+1}\right) \Rightarrow f\left(\vec{\chi}_{i-1}, \psi, \vec{\chi}_{i+1}\right)\right)\right)
$$

to $\Sigma[\mathbf{S B P C}]$, where

$$
f\left(\vec{\chi}_{i-1}, v, \vec{\chi}_{i+1}\right):=f\left(\chi_{1}, \ldots, \chi_{i-1}, v, \chi_{i+1}, \ldots, \chi_{n}\right)
$$

for each $v \in\{\varphi, \psi\}$. SBPC $[\mathcal{F}]$ is called the canonical expansion of SBPC by $\mathcal{F}$-extensional logical connectives. Observe that, by (3.1)$(3.2)$ and $\left(\mathrm{MP}_{\rightarrow}\right), \mathbf{S B P C}[\mathcal{F}]$ has the deduction-detachment theorem (in the sense of [9]) with deduction-detachment set $\{\varphi \rightarrow \psi\}$.

The main result of this announcement asserts 
Theorem 4.1. A deductive system $\mathbf{S}$ over $\mathcal{F}$ is a discriminator logic iff it is definitionally equivalent to an axiomatic extension of the canonical expansion of SBPC by $\mathcal{F}$-extensional logical connectives.

Theorem 4.1 solves the logification problem of Font [22, Section 6.5] for the class of $(\mathbf{0}, \mathbf{1})$-discriminator varieties. A proof of the theorem may be obtained on combining results implicit in [11] with Theorem 3.2.

Let $\mathcal{V}$ be a $(\mathbf{0}, \mathbf{1})$-discriminator variety over $\mathcal{F}$. Another of the main results of [37], which complements Theorem 4.1, yields an effective procedure for axiomatising $S(\mathcal{V}, \mathbf{1})$ as an axiomatic expansion of SBPC by $\mathcal{F}$ extensional logical connectives, given an equational basis for $\mathcal{V}$ and a discriminator term for $\mathcal{V}$ as input. The presentation $\Sigma$ output by the algorithm has the property that its only (proper) rule of inference is $\left(\mathrm{MP}_{\rightarrow}\right)$. Moreover, $\Sigma$ is finite if $\mathcal{V}$ is finitely axiomatisable and $\mathcal{F}$ is finite.

In [11] it is shown that each provability problem $P$ of first-order logic with equality can be effectively reduced to an equivalent provability problem in the equational theory $T_{\mathcal{V}}(P)$ of an appropriate discriminator variety $\mathcal{V}$. Here, the definition of $T_{\mathcal{V}}(P)$ depends on $P$. The variety $\mathcal{G} \mathcal{D}_{(\mathbf{0}, \mathbf{1})}$ always serves in [11] as a starting point for constructing a suitable $\mathcal{V}$, for any given $P$. In [37], an assertional analogue of the reduction of [11] is presented, where $\mathrm{SBPC}$ realises the role of $\mathcal{G} \mathcal{D}_{(\mathbf{0}, \mathbf{1})}$ in constructing a logical cognate of $\mathcal{V}$. In the reduction of [37], the first-order logical connectives $\cap, \cup, \supset$, and $\sim$ are replaced with the propositional connectives $\wedge, \vee, \rightarrow$, and $\neg$ respectively of SBPC, while the equality symbol $\approx$ is replaced with the symmetrisation of the propositional connective $\Rightarrow$.

\section{$5 \quad \mathrm{~S} 5$ as a discriminator logic}

To illustrate Theorem 4.1, we present the normal modal logic S5 ("Gödel style") as an axiomatic expansion of SBPC by extensional logical connectives.

Let $\Lambda\left[\mathbf{S} 5^{G}\right]:=\Lambda[\mathbf{C P C}] \cup\{\square\}$, where $\square$ is a unary logical connective (the necessity sign). Let $\mathbf{S} 5^{G}$ denote the normal modal logic $\mathbf{S} 5$ in the "Gödel style" formulation of $\left[9\right.$, Section 2.2.5] over the language $\Lambda\left[\mathbf{S} 5^{G}\right]$. (Here, the rule of necessitation is taken in its strong form: $\varphi \vdash \square \varphi .^{1}$ For a discussion and references, see [24, Section 2.4, p. 57].)

Let $\mathbf{S}$ denote the deductive system over the language $\Lambda[\mathbf{S}]:=\Lambda[\mathbf{S B P C}] \cup$ $\Lambda\left[\mathbf{S 5}^{G}\right]$ determined by the axioms and inference rules of $\Sigma[\mathbf{S B P C}]$, the ax-

\footnotetext{
${ }^{1}$ This implies that the modal consequence relation $\vdash_{\mathbf{S 5}^{G}}$ is global in the sense of [33, Section 3.2], and $\mathbf{S 5}{ }^{G}$ is algebraisable in the sense of [7].
}

Australasian Journal of Logic (11:2) 2014, Article no. 5 
ioms (only) of $\Sigma[\mathbf{C P C}]$, the compatibility axioms (4.1) for each $n$-ary logical connective $f \in \Lambda\left[\mathbf{S} 5^{G}\right]$, together with the 'link' axioms

$$
\begin{aligned}
& \vdash \neg(\varphi \Rightarrow \varphi) \supset \varphi \\
& \vdash((\varphi \supset \psi) \supset \psi) \Rightarrow((\psi \supset \varphi) \supset \varphi) \\
& \vdash(\varphi \supset \psi) \Rightarrow(\varphi \rightarrow \psi) .
\end{aligned}
$$

Observe that, by (5.1), modus ponens for $\supset$ is a derived rule of inference of $\mathbf{S}$. Hence $\vdash_{\mathbf{C P C}} \subseteq \vdash_{\mathbf{S}}$.

\section{Theorem 5.1.}

1. The map $\delta: \Lambda[\mathbf{S}] \rightarrow \mathrm{Fm}_{\Lambda\left[\mathbf{S} \mathbf{5}^{G}\right]}$ defined by

$$
\begin{array}{rlrl}
p \cap q & \mapsto p \cap q & & \wedge q \mapsto p \cap(\square p \supset q) \\
p \cup q & \mapsto p \cup q & p \vee q & \mapsto \square p \cup q \\
\sim p & \mapsto \sim p & & \neg p \mapsto \square p \supset \sim(p \supset p) \\
p \supset q & \mapsto p \supset q & p & \rightarrow q \mapsto \square p \supset q \\
& p \Rightarrow q \mapsto \square((p \supset q) \cap(q \supset p)) \cup q
\end{array}
$$

is an interpretation of $\mathbf{S}$ in $\mathbf{S} 5^{G}$.

2. The map $\epsilon: \Lambda\left[\mathbf{S 5}^{G}\right] \rightarrow \mathrm{Fm}_{\Lambda[\mathbf{S}]}$ defined by

$$
\begin{array}{rlrl}
p \cap q \mapsto p \cap q & \sim p & \mapsto \sim p \\
p \cup q \mapsto p \cup q & p \supset q & \mapsto p \supset q \\
& \square p & \mapsto(p \rightarrow \neg(p \Rightarrow p)) \Rightarrow \neg(p \Rightarrow p)
\end{array}
$$

is an interpretation of $\mathbf{S} 5^{G}$ in $\mathbf{S}$.

3. The interpretations $\delta$ and $\epsilon$ are mutually inverse.

Hence the deductive systems $\mathbf{S}$ and $\mathbf{S} 5^{G}$ are definitionally equivalent.

Theorem 5.1 shows that $\mathbf{S 5}{ }^{G}$ is in some sense obtained by 'gluing' CPC and SBPC together.

The preceding observation can be made precise on interpreting Theorem 5.1 algebraically. Let $\mathcal{B} \mathcal{A}$ denote the class of all Boolean algebras $\langle A ; \cap, \cup, \sim, 0,1\rangle$, and let $\mathcal{B A}^{t}$ denote the class obtained from $\mathcal{B} \mathcal{A}$ by adjoining a new ternary function symbol $t$ to the language of $\mathcal{B} \mathcal{A}$ such that the realisation of $t$ on any member of $\mathcal{B A}$ is the ternary discriminator. Theorem 5.1 asserts that the algebraic counterpart of $\mathbf{S} 5^{G}$ (viz., the variety of monadic algebras) is, up to term equivalence, precisely the variety generated by $\mathcal{B} \mathcal{A}^{t}$. This observation has apparently been known to specialists in general algebra for some time. (See, for example, [12, Corollary 2.7].) 


\section{Concluding remarks}

The main result of this research announcement extends, mutatis mutandis, to several generalisations of the class of discriminator logics, the most important of which is the class of 'positive fixedpoint discriminator logics'. A deductive system $\mathbf{S}$ is a positive fixedpoint discriminator logic if $\mathbf{S}=S(\mathcal{V}, \mathbf{1})$ for a fixedpoint 1-discriminator variety $\mathcal{V}$. For the concept of a fixedpoint 1-discriminator variety, see [8].

Let $\Lambda[\mathbf{B C S K}]$ denote the language consisting of the binary logical connectives $\Rightarrow$ (the strong implication sign) and $\rightarrow$ (the weak implication sign), and let $\mathcal{F}$ be a language with $\mathcal{F} \cap \Lambda[\mathbf{B C S K}]=\emptyset$. Let BCSK denote the deductive system over $\Lambda$ [BCSK $]$ presented by those axioms and inference rules of $\Sigma[$ SBPC] not involving any of the connectives $\wedge, \vee$, and $\neg$ and let the canonical expansion of BCSK by $\mathcal{F}$-extensional logical connectives be defined in the same way as for SBPC $[\mathcal{F}]$; for studies of BCSK, see $[27,28]$. For positive fixedpoint discriminator logics, the analogue of Theorem 4.1 is

Theorem 6.1. A deductive system $\mathbf{S}$ over $\mathcal{F}$ is a positive fixedpoint discriminator logic iff it is definitionally equivalent to an axiomatic extension of the canonical expansion of BCSK by $\mathcal{F}$-extensional logical connectives.

Theorem 6.1 solves the logification problem of Font [22, Section 6.5] for the class of fixedpoint 1-discriminator varieties.

The $\{\supset, \square\}$-fragment of $\mathbf{S} 5^{G}$ constitutes a natural example of a deductive system that is a positive fixedpoint discriminator logic, but not a discriminator logic; numerous other examples may be found in the literature.

Let $\mathbf{B C S K}_{\neg}$ denote the axiomatic expansion of BCSK by a unary logical connective $\neg$ (the negation sign) and those axioms of $\Sigma[$ SBPC] involving $\neg$. One of the remaining technical results of [37] shows that $\mathbf{B C S K}_{\neg}$ is definitionally equivalent to its own 'anti-logic', wherein the theorems [resp. contradictions] of the former are precisely the contradictions [resp. theorems] of the latter, and conversely. It is shown in [37] that this 'anti-logic' has a (comparatively) familiar description: it is a modalised analogue of the hoop logic $\mathbf{S}_{\mathcal{H O}}$ of [6]. The logic $\mathbf{S}_{\mathcal{H O}}$ is itself a natural generalisation of the conjunction-implication fragment of the intuitionistic propositional calculus; for details, see $[6,8]$.

\section{References}

[1] M. Baaz, 'Infinite-valued Gödel logic with 0-1-projections and relativisations', in Gödel'96: Logical Foundations of Mathematics, Computer 
Science, and Physics (P. Hájek, ed.), Lecture Notes in Logic, vol. 6, Springer-Verlag, Brno, 1996, pp. 23-33.

[2] H. Barringer, J. H. Cheng, and C. B. Jones, A logic covering undefinedness in program proofs, Acta Inform. 21 (1984), 251-269.

[3] V. Boicescu, A. Filipoiu, G. Georgescu, and S. Rudeanu, LukasiewiczMoisil algebras, North-Holland, Amsterdam, 1991.

[4] R. J. Bignall and J. E. Leech, Skew Boolean algebras and discriminator varieties, Algebra Universalis 33 (1995), 387-398.

[5] U. Blau, Die dreiwertige Logik der Sprache, Grundlagen der Kommunikation, Walter de Gruyter, Berlin, 1978.

[6] W. J. Blok and I. M. A. Ferreirim, On the structure of hoops, Algebra Universalis 43 (2000), 233-257.

[7] W. J. Blok and D. Pigozzi, Algebraizable Logics, Mem. Amer. Math. Soc., no. 396, American Mathematical Society, Providence, 1989.

[8] _ On the structure of varieties with equationally definable principal congruences III, Algebra Universalis 32 (1994), 545-608.

[9] - Abstract algebraic logic and the deduction theorem, manuscript (available from http://orion.math.iastate.edu/dpigozzi/papers/ aaldedth.pdf, accessed 7 December 2013), 2001.

[10] W.J. Blok and J.G. Raftery, Assertionally equivalent quasivarieties, Internat. J. Algebra Comput. 18 (2008), 589-681.

[11] S. Burris, Discriminator varieties and symbolic computation, J. Symbolic Comput. 13 (1992), 175-207.

[12] S. Burris, R. McKenzie, and M. Valeriote, Decidable discriminator varieties from unary varieties, J. Symbolic Logic 56 (1991), 1355-1368.

[13] S. Burris and H. P. Sankappanavar, A Course in Universal Algebra, Graduate Texts in Mathematics, no. 78, Springer-Verlag, New York, 1981.

[14] C. Caleiro and R. Gonçalves, 'Equipollent logical systems', in Logica Universalis: Towards a General Theory of Logic, (J.-Y. Beziau, ed.), Birkhäuser Verlag, Basel, 2005, pp. 99-111. 
[15] G. Cattaneo, D. Ciucci, R. Giuntini, and M. Konig, Algebraic structures related to many-valued logical systems. Part I: Heyting-Wajsberg algebras, Fund. Inform. 63 (2004), 331-355.

[16] _ Algebraic structures related to many-valued logical systems. Part II: Equivalence among some widespread structures, Fund. Inform. 63 (2004), 357-373.

[17] J. Czelakowski, Protoalgebraic logics, Trends in Logic (Studia Logica Library), vol. 10, Kluwer, Dordrecht, 2001.

[18] J. Czelakowski and D. Pigozzi, Fregean logics, Ann. Pure Appl. Logic 127 (2004), 17-76.

[19] G. Domenech, H. Freytes, and C. de Ronde, Scope and limits of modality in quantum mechanics, Ann. Physik 15 (2006), 853-860.

[20] , Modal-type orthomodular logic, Math. Log. Quart. 55 (2009), $307-319$.

[21] G. Epstein and A. Horn, Logics which are characterised by subresiduated lattices, Zeitschr. Math. Logik Grundlagen Math. 22 (1976), 199-210.

[22] J. M. Font, 'Generalized matrices in abstract algebraic logic', in Trends in logic: 50 years of Studia Logica (V. Hendriks and J. Malinowski, eds.), Trends in Logic (Studia Logica Library), vol. 21, Kluwer Academic Publishers, Dordrecht, 2003, pp. 57-86.

[23] J. M. Font and M. Rius, An abstract algebraic logic approach to tetravalent modal logics, J. Symbolic Logic 65 (2000), 481-518.

[24] J. M. Font and R. Jansana, A General Algebraic Semantics for Sentential Logics (2nd edn.), Lecture notes in Logic, vol. 7, Association for Symbolic Logic, 2009.

[25] V. Gyuris, Variations of Algebraizability, Ph.D. thesis, The University of Illinois at Chicago, 1999.

[26] P. Hájek, Metamathematics of fuzzy logic, Trends in Logic (Studia Logica Library), vol. 4, Kluwer Academic Publishers, Dordrecht, 1998.

[27] L. Humberstone, An intriguing logic with two implicational connectives, Notre Dame J. Formal Logic 41 (2000), 1-41.

Australasian Journal of Logic (11:2) 2014, Article no. 5 
[28] _ Identical twins, deduction theorems, and pattern functions: Exploring the implicative BCSK fragment of $\boldsymbol{S 5}$, J. Philos. Logic 35 (2006), 435-487.

[29] L. Itturioz, 'Modal operators on symmetrical Heyting algebras', in Universal Algebra and Applications, Banach Centre Publications, vol. 9, Institute of Mathematics, Polish Academy of Sciences, Warszawa, 1982, pp. 289-303.

[30] L. Itturioz and E. Orłowska, A Kripke-style and relational semantics for logics based on Eukasiewicz algebras, J. Multiple-Valued Logic and Soft Comput. 12 (2006), 131-147.

[31] C. B. Jones, Systematic Software Development Using VDM, PrenticeHall International, United Kingdom, 1986.

[32] T. Kowalski, Varieties of tense algebras, Rep. Math. Logic 32 (1998), 53-95.

[33] M. Kracht, 'Modal consequence relations', in Handbook of Modal Logic (J. van Benthem, P. Blackburn, and F. Wolter, eds.), Studies in Logic and Practical Reasoning, vol. 3, Elsevier, 2006, pp. 491-545.

[34] R. McKenzie, G. F. McNulty, and W. F. Taylor, Algebras, Lattices, Varieties, vol. 1, Wadsworth \& Brooks/Cole, Monterey, 1987.

[35] T. Smiley, The independence of connectives, J. Symbolic Logic 27 (1962), 426-436.

[36] M. Spinks and R. Veroff, Constructive logic with strong negation is a substructural logic. II, Studia Logica 89 (2008), 401-425.

[37] M. Spinks, R. J. Bignall, and R. Veroff, Discriminator logics, in preparation.

[38] R. Suszko, Ontology in the Tractatus of L. Wittgenstein, Notre Dame J. Formal Logic 9 (1968), 7-33.

[39] _ Investigations into the sentential calculus with identity, Notre Dame J. Formal Logic 13 (1972), 289-308.

[40] _ 'Abolition of the Fregean axiom', in Logic Colloquium (Symposium on Logic Held at Boston, 1972-73) (R. Parikh, ed.), Lecture Notes in Mathematics, vol. 453, Springer-Verlag, Berlin, 1975, pp. 169-239. 
[41] R. Veroff and M. Spinks, Axiomatizing the skew Boolean propositional calculus, J. Automated Reasoning 37 (2006), 3-20.

Australasian Journal of Logic (11:2) 2014, Article no. 5 